\title{
Excitability transitions and wave dynamics under spatiotemporal structured noise
}

\author{
S. Alonso, ${ }^{1,2, *}$ F. Sagués, ${ }^{1}$ and J. M. Sancho ${ }^{2}$ \\ ${ }^{1}$ Departament de Química Física, Universitat de Barcelona, Avenida Diagonal 647, 08028 Barcelona, Spain \\ ${ }^{2}$ Departament d'Estructura i Constituents de la Matèria, Universitat de Barcelona, Avenida Diagonal 647, 08028 Barcelona, Spain
}

(Received 16 October 2001; published 14 June 2002)

\begin{abstract}
We present an analytic and numerical study of the effects of external fluctuations in active media. Our analytical methodology transforms the initial stochastic partial differential equations into an effective set of deterministic reaction-diffusion equations. As a result we are able to explain and make quantitative predictions on the systematic and constructive effects of the noise, for example, target patterns created out of noise and traveling or spiral waves sustained by noise. Our study includes the case of realistic noises with temporal and spatial structures.
\end{abstract}

DOI: 10.1103/PhysRevE.65.066107

PACS number(s): 82.40.Bj, 05.40.-a, 47.54.+r

\section{INTRODUCTION}

Excitability, in relation to wave propagation processes, is one of the most genuine features of distributed active media $[1,2]$, irrespective of the context we refer to, either chemical [3], biological [4], cardiological [5], or neurophysiological [6]. In fact, we could think of excitability as one of the main robust mechanisms to sustain signal propagation in nature. The diversity of such self-organized propagating patterns varies largely, depending on initial conditions or geometrical constraints: from the simplest one-dimensional wave fronts [7] to spiral waves [8] and target patterns [9] in twodimensional systems, or adopting more complicated topologies, such as scroll waves and scroll rings [10] in threedimensional media.

Obviously, in any realistic situation we can envisage, active media cannot be completely isolated from their environment. Thus, the control, either global or local, of the excitability of a given medium will be unavoidably subjected to imperfections or fluctuations. Until recently, and most commonly, one would consider this statement as a sort of word of caution to prevent experimentalists or to discourage theoreticians. However, during this last decade a new twist on this generally accepted idea has been progressively emerging after the discovery of more and more subtle examples of what are presently viewed as noise-constructive effects in nonlinear systems [11]. In this paper we will show abundant examples of phenomenologies occurring in excitable media that can be addressed under this general perspective.

To gain the widest possible universality we propose a generic theoretical framework that covers the whole spectrum of excitability conditions considered here: from nonexcitable (media unable to propagate any kind of pulses) to subexcitable (contraction of waves with free ends) up to excitable and even to oscillatory regimes. In doing so our goal is twofold: (i) First, we want to demonstrate that some previous observations in noisy excitable systems, both experimental and numerical, concerning photosensitive versions of the Belousov-Zhabotinsky reaction mainly by Kádár et al. [12] and by us [13], are in fact particular examples, with addi-

\footnotetext{
*Corresponding author. Email address: s.alonso@qf.ub.es
}

tional ones furnished here, of what we denote as noiseinduced excitability transitions. (ii) Our second purpose is to extract robust analytical and numerical evidences of the profound effect of external fluctuations coupled to the nonlinear dynamics of excitable systems to ultimately modify their wave propagation properties. Actually we want to prove that this is not at all a specific effect associated to some peculiar statistical prescriptions of the imposed fluctuations. In this respect we extend our previous research [13], where we focused on purely uncorrelated partitioned fluctuations (white noise) to address more realistic situations corresponding to spatiotemporal structured random forcing of the active media. What comes out of our study, and this is the main generic conclusion we want to stress, is that such spatiotemporal structured noise forcing, although locally and globally averaging to zero, always enhances the medium excitability, favoring in turn wave propagation conditions.

Unlike our previous related papers [14] and [13], we prefer here to concentrate on the theoretical treatment of the problem, without accompanying experiments, but supplementing it with extensive numerical simulations. The theoretical part is totally generic as it would apply to FitzHughNagumo-like schemes to describe active systems. Specific applications of these theoretical results, whenever needed, and particularly the whole numerical part of the paper will refer, however, to the simplest modelization of such excitable dynamics, namely, the Barkley's model [15]

$$
\begin{gathered}
\partial_{t} u=D \nabla^{2} u+\frac{1}{\epsilon} u(1-u)\left(u-\frac{v+b}{a}\right), \\
\partial_{t} v=u-v .
\end{gathered}
$$

After these introductory remarks, the paper is organized as follows. Section II is devoted to present the theoretical treatment that enables us to deal with excitable systems subjected to spatiotemporal structured fluctuations. Section III summarizes the different scenarios of what was denoted previously as noise-induced excitability transitions. To highlight the main trends of these phenomena we prefer to limit at this point to white (temporally uncorrelated) fluctuations stressing the effect of the intensity of the fluctuations. The complete analysis, including correlations both in space and time, 
is concentrated in Sec. IV. The paper ends with a Conclusion section and an Appendix where we compile the technical details necessary to conveniently handle spatiotemporally correlated fluctuations.

\section{STOCHASTIC MODELIZATION AND EFFECTIVE DETERMINISTIC MODEL}

Although as mentioned above explicit results will be obtained for the Barkley's model, we adopt on what follows a more general perspective by formulating our analytical treatment as it would apply to a generic active medium described by an activator-inhibitor (FitzHugh-Nagumo-like) dynamics,

$$
\begin{gathered}
\partial_{t} u=D \nabla^{2} u+f(u, v ; b), \\
\partial_{t} v=u-v .
\end{gathered}
$$

Note that, as usual in this context, diffusion of the inhibitor has been neglected and, for the sake of simplicity, we have incorporated the time scale parameter of the model into the generic definition of the kinetic term. In the equations above $b$ is the parameter that is assumed to control the excitability of the medium, i.e., it would represent, for example, the intensity of light in the photosensitive BelousovZhabotinsky (BZ) reaction. It is thus natural to introduce external fluctuations acting on the medium through this parameter by letting it to fluctuate according to $b \rightarrow b$ $+\eta(\mathbf{x}, t)$ (to avoid overloading notation we keep on denoting the mean value with the same symbol $b$ ). In this way we arrive at the following generic system of stochastic partial differential equations (SPDE),

$$
\begin{gathered}
\partial_{t} u=D \nabla^{2} u+f(u, v, b)+g(u) \eta(\mathbf{x}, t), \\
\partial_{t} v=u-v .
\end{gathered}
$$

In fact, the only implicit limitation to what we have committed ourselves when writing Eq. (3), and certainly valid for the Barkley's model, is that the noise enters directly only in the dynamics of the activator variable, and, furthermore, we assume that it is coupled explicitly only with this variable. Although this in not in any way a fundamental requirement of our theoretical analysis, it renders its practical handling much simpler, as exemplified on what follows. The only remaining task is to specify the statistical properties of the fluctuating force $\eta(\mathbf{x}, t)$. We prescribe it as a Gaussian spatiotemporal distributed noise with zero mean and correlation given by

$$
\begin{aligned}
\left\langle\eta\left(\mathbf{x}^{\prime}, t^{\prime}\right) \eta(\mathbf{x}, t)\right\rangle & =G\left(\left|\mathbf{x}-\mathbf{x}^{\prime}\right|,\left|t-t^{\prime}\right|\right) \\
& =C\left(\left|\mathbf{x}-\mathbf{x}^{\prime}\right| / \lambda\right) \gamma\left(\left|t-t^{\prime}\right| / \tau\right),
\end{aligned}
$$

where for a $d$-dimensional system,

$$
C(r / \lambda)=\frac{\sigma^{2}}{(\sqrt{2 \pi})^{d} \lambda^{d}} e^{-r^{2} / 2 \lambda^{2}}
$$

$$
\gamma(s / \tau)=\frac{1}{\tau} e^{-s / \tau}
$$

For the sake of simplicity we have adopted isotropic, stationary, and uniform properties. In addition we propose a decoupling ansatz for the temporal and spatial dependences. In this way we highlight the role of the three basic input parameters of the external fluctuations: intensity $\left(\sigma^{2}\right)$, correlation time $(\tau)$, and correlation length $(\lambda)$ [16].

The set of equations (3)-(5) does not admit an exact solution. The important point to realize, however, is that due to the multiplicative nature of the random term, one can separate its systematic effects from those that trivially average to zero. Moreover, we will show how to transfer the former into an effective deterministic model statistically equivalent to the original SPDE set. Note in passing that the assumption of such a multiplicative character of the fluctuations is by no means unreasonable, since commonly, and singularly for realistic Oregonator-like models of the BZ reactions, activatorinhibitor simplified descriptions of active media do result from adiabatic reduction procedures of more complete reaction-diffusion schemes originally containing additive fluctuations [17].

The practical recipe to extract such systematic contributions is the following. We denote the nonzero mean value of the noisy term,

$$
\langle g(u(\mathbf{x}, t)) \eta(\mathbf{x}, t)\rangle \equiv\langle\Phi(u)\rangle \neq 0 .
$$

By adding and substracting $\Phi(u)$ to the equation of motion for $u$ in Eq. (3) we arrive at

$$
\begin{gathered}
\partial_{t} u=D \nabla^{2} u+f(u, v, b)+\Phi(u)+R(u ; \mathbf{x}, t), \\
\partial_{t} v=u-v,
\end{gathered}
$$

where $R(u ; \mathbf{x}, t)=g(u) \eta(\mathbf{x}, t)-\Phi(u)$ is a more complicated random term but has a zero mean value and whose effects can be safely discarded for not very large noise intensities. The original SPDE set is thus equivalent to an effective deterministic model, formally expressed as

$$
\begin{gathered}
\partial_{t} u=D \nabla^{2} u+f(u, v, b)+\Phi(u), \\
\partial_{t} v=u-v .
\end{gathered}
$$

Following the steps derived in Ref. [17] we have that

$$
\Phi(u)=\phi_{0}(u)+\left[\phi_{1 a}(u)+\phi_{1 b}(u)+\phi_{1 c}(u)\right] \tau+\cdots,
$$

where $\phi_{0}$ stands for the white noise systematic contribution, and $\phi_{1 i}$ denotes the first order contributions of an expansion based on the correlation time $\tau$. Explicitly,

$$
\begin{gathered}
\phi_{0}(u)=C(0) g(u) g^{\prime}(u), \\
\phi_{1 a}(u)=-C(0) g^{\prime}(u)\{g(u), f(u, v)\}, \\
\phi_{1 b}(u)=C(0) D g^{\prime}(u) g^{\prime \prime}(u)(\nabla u)^{2},
\end{gathered}
$$




$$
\phi_{1 c}(u)=D C^{\prime \prime}(0) g(u) g^{\prime}(u)
$$

where $\{g, f\}=g^{\prime} f-g f^{\prime}$, and the derivatives are taken with respect to the activator field $u$. We remark here that the dependence on the correlation length $\lambda$ is included through $C(0)$ and $C^{\prime \prime}(0)$ (see Sec. IV).

The explicit form of these contributions can be obtained readily for the model Eq. (1). The first term $\phi_{0}(u)$ is the standard Stratonovich's contribution [16] and the $\phi_{1 c}(u)$ term is formally equal to $\phi_{0}(u)$, so we explicitly obtain

$$
\begin{gathered}
\phi_{0}(u)=\frac{C(0)}{a^{2} \epsilon^{2}} u(1-u)(1-2 u), \\
\phi_{1 c}(u)=\frac{D C^{\prime \prime}(0)}{a^{2} \epsilon^{2}} u(1-u)(1-2 u) .
\end{gathered}
$$

The $\phi_{1 a}(u)$ contribution is written as

$$
\phi_{1 a}(u)=\frac{C(0)}{a^{2} \epsilon^{3}} u^{2}(1-u)^{2}(1-2 u)
$$

Here a higher-order polynomial appears whose influence on the wave dynamics is impossible to determine analytically. Our practical strategy here consists in substituting it for an equivalent and simpler functional form like those appearing in Eqs. (12) and (13). As can be readily checked this can be accomplished through the simple introduction of a numerical prefactor $\beta=\frac{6}{25}(\sqrt{3} / \sqrt{5})$ [see Fig. 1(a)]. This prefactor has been calculated imposing the same maximum value for the two polynomials $\left[u^{2}(1-u)^{2}\right.$ and $\left.\beta u(1-u)\right]$ in the interval $[0,1]$.

Thus, the contribution of Eq. (14) is replaced by the appropriate expression:

$$
\phi_{1 a}(u) \approx \frac{C(0)}{a^{2} \epsilon^{3}} \beta u(1-u)(1-2 u) .
$$

Finally, for the treatment of the term containing spatial derivatives $\phi_{1 b}(u)$,

$$
\phi_{1 b}(u)=-\frac{C(0) D}{a^{2} \epsilon^{2}} 2(1-2 u)(\nabla u)^{2},
$$

we perform numerical derivatives of the one-dimensional profile for the activator variable with the ultimate purpose to reduce the whole contribution to the same functional form of the previously evaluated terms [see Fig. 1(b)]. Skipping the details, we end up with

$$
\phi_{1 b}(u)=-\frac{C(0)}{a^{2} \epsilon^{2}} 2 D \gamma u(1-u)(1-2 u),
$$

where $\gamma \approx 12$.

Finally, by putting all these contributions together, we get
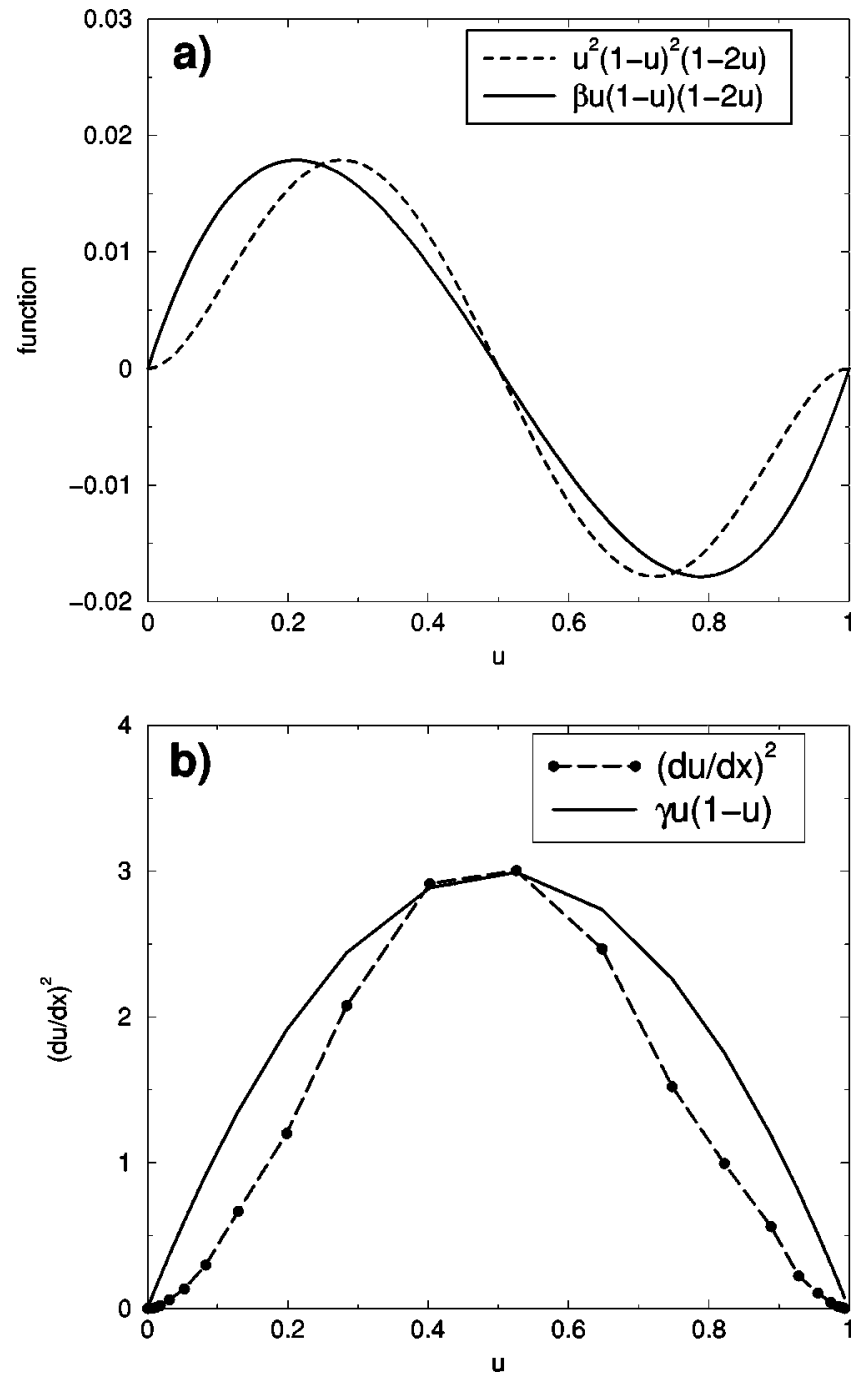

FIG. 1. (a) Plot of the functionality of Eq. (14) (dotted line) and the approximation used in Eq. (15) (solid line). (b) Square of the second spatial derivate of the activator variable (dashed line). Approximation proposed in Eq. (17) with $\gamma \approx 12$ (solid line).

$$
\phi_{1}(u) \tau=-\frac{C(0) u(1-u)(1-2 u)}{a^{2} \epsilon^{2}} \frac{\tau}{\tau_{0}},
$$

where we have introduced a new time scale $\tau_{0}$ defined as

$$
\tau_{0}^{-1}(\lambda)=-\frac{D C^{\prime \prime}(0)}{C(0)}-\frac{\beta}{\epsilon}+2 D \gamma
$$

This is one of the relevant parameters of our analysis, which for realistic choices of the parameter values is positive. It contains three terms each one being a characteristic time. The most important for us is the first one because it keeps the dependence on the noise correlation length $\lambda$, being independent of $\sigma^{2}$ and $\tau$. The other two terms are associated with the time scale of the reaction and diffusion, respectively.

Using these final expressions we arrive at an effective and renormalized version of Barkley's model [Eq. (1)], with the following parameter redefinitions: 

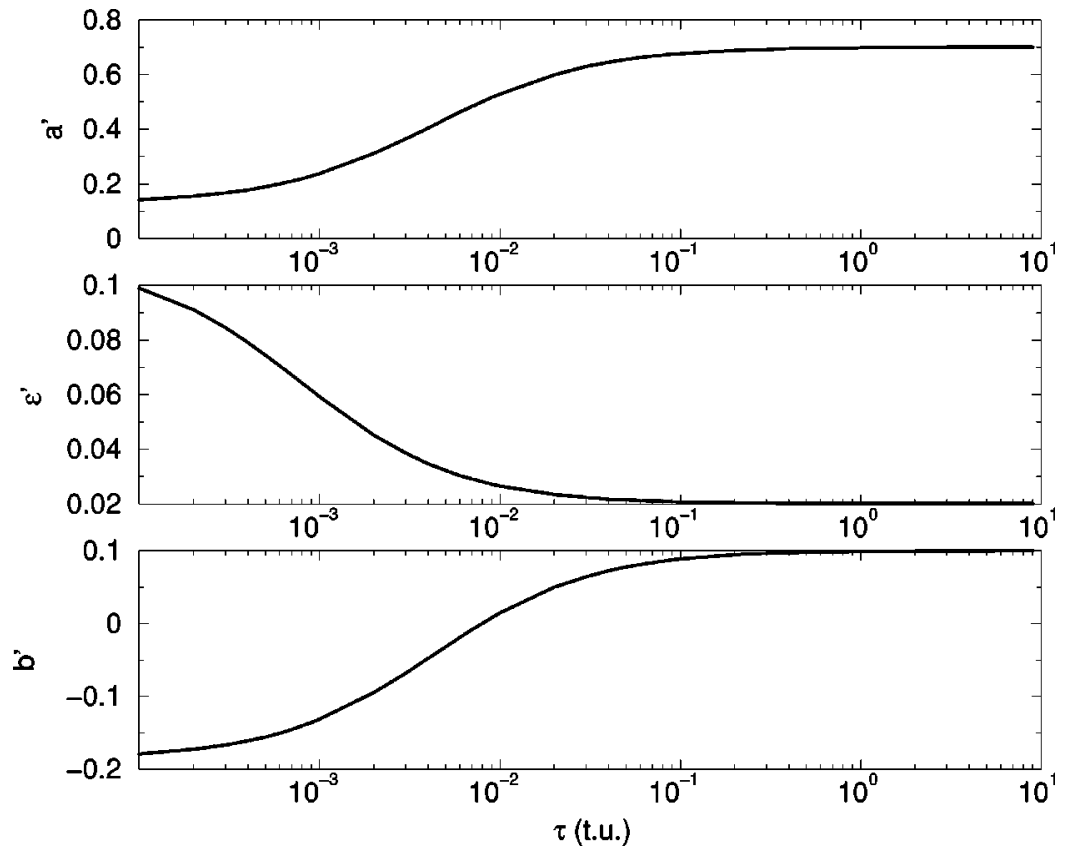

FIG. 2. Parameters of the effective model Eqs. (23)-(25) vs the temporal correlation of the noise $(\tau)$. In this and following figures, the symbols s.u. and t.u. correspond, respectively, to the dimensionless spatial and temporal units used for the model in Eq. (1). Parameter values are $a$ $=0.7, \quad b=0.1, \quad \epsilon=0.02, \quad D=1, \quad C(0)=0.004$, $C^{\prime \prime}(0)=-0.8$, and $\tau_{0}=0.0047$ t.u.

$$
\begin{gathered}
a^{\prime}=a\left(1-\frac{2}{\epsilon a^{2}} C(0)\left(1-\tau / \tau_{0}\right)\right), \\
b^{\prime}=b-\frac{1}{\epsilon a} C(0)\left(1-\tau / \tau_{0}\right), \\
\epsilon^{\prime}=\frac{\epsilon}{\left[1-\frac{2}{\epsilon a^{2}} C(0)\left(1-\tau / \tau_{0}\right)\right]}
\end{gathered}
$$

Although we admit that this particularly simple renormalization scheme is model dependent, the procedure is quite general and its predictions are quite robust, as shown in following sections, when they are statistically compared with numerical simulations of the original stochastic model.

It is clear that these results have been obtained for small noise intensities and temporal correlations and therefore some inconsistencies should be expected for large values of these parameters. In order to get a better analytical convergence we further propose a standard analytic regularization of the previous first-order expressions. The simplest regularization of the $\tau$ dependence, giving the deterministic limit when $\tau \rightarrow 0$, would be expressed by (see Fig. 2)

$$
\begin{gathered}
a^{\prime}=a\left(1-\frac{2 C_{R}(0)}{\epsilon a^{2}}\right), \\
b^{\prime}=b-\frac{C_{R}(0)}{\epsilon a}, \\
\epsilon^{\prime}=\frac{\epsilon}{\left(1-\frac{2 C_{R}(0)}{\epsilon a^{2}}\right)},
\end{gathered}
$$

where for simplicity we have introduced the effective parameter $C_{R}(0)$ into the renormalized expressions for the model parameters,

$$
C_{R}(0)=\frac{C(0)}{\left[1+\tau / \tau_{0}(\lambda)\right]} .
$$

This is another relevant effective parameter in our analytical approach. It can be understood as a renormalization of the noise intensity, which incorporates the influence of the three parameters of the noise $\left[\right.$ remember that $\left.C(0) \sim \sigma^{2} / \lambda^{d}\right]$. The white noise intensity is trivially recovered for $\tau \rightarrow 0$ leading to the well-known Stratonovich contribution, and the deterministic limit is obtained as $\sigma^{2} \rightarrow 0, \tau \rightarrow \infty$, or $\lambda \rightarrow \infty$.

The behavior of $\tau_{0}$ and $C_{R}(0)$ as a function of the noise parameters will help us in the interpretations and predictions relevant to the phenomena in noisy excitable systems reported in the following sections.

\section{NOISE-INDUCED EXCITABILITY TRANSITIONS}

Quite diverse are the wave propagation phenomenologies that can be found in spatially extended active media. In this section we focus on the most paradigmatic examples, i.e., single front propagation (either with or without free ends), spiral waves or target patterns. Our goal at this point is to show that external distributed fluctuations can be effectively used to tune the global excitability properties of the medium, and in turn modify the conditions under which the different regimes of wave propagation are observed. Although such an effect will naturally depend on the different parameters of the fluctuations, i.e, their intensity and length and time correlations, we will restrict ourselves in this section to the consideration of noncorrelated, neither temporal nor spatial, noise. In this way, we hope to stress the generic nature of what we have called noise-induced excitability transitions and, at the same time, focus on the role of the fluctuation intensity as 
the main parameter controlling such transitions.

All the numerical simulations reported in this section thus correspond to run implemented discretized codes for numerical integration, via an implicit Heun method, of the original SPDE adapted to the Barkley's model. This is done once the spatially distributed fluctuations are prescribed through independent realizations on each grid cell of a Gaussian white random process. To compare with our analytical predictions, we only need to run simulations of the deterministic effective model with the model parameters renormalized according to the results of the preceding section, Eqs. (23)-(26), under the limit $\tau \rightarrow 0$ and with intensity $C(0)=\sigma^{2} /(\Delta x)^{d}$.

\section{A. Nonexcitable-excitable transition: Noise-supported wave propagation}

The simplest case to begin with corresponds to onedimensional propagation conditions, when active media are unable to support waves (nonexcitable regime). Let us suppose now that to such a nonexcitable medium we simply superimpose distributed uncorrelated fluctuations that average to zero. As repeatedly stated above, we modify in this way the global excitability properties of the medium and as a result we do observe in this case steadily propagating waves. This is illustrated in Fig. 3 for circular fronts, where respectively we plot propagation conditions of the fluctuation-free nonexcitable medium (first column in Fig. 3), of the noisy forced situation (second column in Fig. 3), and finally, for the sake of comparison, of the effective deterministic system (third column in Fig. 3).

A somewhat more quantitative study of such a nonexcitable-excitable transition, this time as it applies to strictly one-dimensional fronts, is summarized in Fig. 4. Taking the velocity propagation of stable fronts as indicator, we can neatly identify the intensity-dependent fluctuation threshold for one-dimensional noise-sustained wave propagation. Also note the monotonous increase of the front velocity, as the noise intensity increases, as a direct signature of the excitability enhancement in the medium. We stress the very good agreement we observe between the simulations of the noisy system and those for the deterministic effective one. In the former case, obviously, results come from averages among several realizations, the velocity being taken at the very first time the front is formed.

More spectacular results are expected to occur in going to real two-dimensional propagation conditions. In this context, a pair of situations will be considered in turn: spiral waves and target patterns.

\section{B. Subexcitable-excitable transition: Noise-sustained spirals}

The control of shrinking or sprouting of autowaves with free ends, as considered previously in other experimental or numerical situations $[12,13]$, appears to be a perfect scenario to be addressed from the point of view of the effect of external fluctuations on excitable media. The most crucial example corresponds here to an initially subexcitable medium for which an initially created spiral wave retraces back and finally disappears. Similarly to what was reported in the preceding section, by simply adding distributed uncorrelated fluctuations, we do sustain in this case steady rotating spirals that rapidly adopt a characteristic pitch and rotation period after a few rotations. Numerical simulations are plotted in Fig. 5, organized similarly to the previous considered case. Actually, as we can see in Fig. 5, our effective model is not only able to reproduce the final pitch of the spiral under fluctuations, but also its transient behavior to adopt a steady rotation mode.

Again, a somewhat more quantitative analysis of such a subexcitable-excitable transition, was conducted by referring to one of the characteristic parameters of the stable rotating waves, in this case the rotation period. Results are summarized in Fig. 6 for an intermediate range of noise intensities before spiral breakup induced by noise [18]. It is clear in this case, again related to the noise-enhanced excitability property, that the rotation period decreases with the fluctuation intensity. With reference to Fig. 6, the boundary between subexcitable and excitable conditions is numerically fixed as the condition of expanding versus contracting a wave with a free end, this being the condition of spiral formation in an infinite medium. In passing we justify in this way, the shift of our finite size simulation results. Another numerical difficulty we meet and that could explain also some discrepancies, is the Brownian motion of the spiral tip [14], which renders the practical computation of the rotation period a somewhat difficult task, singularly for weak excitability.

\section{Excitable-oscillatory transition: A pacemaker created out of noise}

Actually this is the situation that motivated our experimental and numerical research previously published on noise-induced excitability transitions [13]. We start in this case with an excitable medium perturbed with a circular wave front that expands, without reproducing itself as expected, to encompass the whole system. By randomly forcing the excitability of the medium, however, not only the front waves move faster but more importantly, repeat themselves to, transiently, create a pacemaker regularly emitting waves. Numerical simulations are plotted in Fig. 7, organized similarly to the previous cases. It is worth remarking at this point that our observations, in accordance with theoretical predictions, certainly indicate that in most cases the regular target pattern structure is not stable, being eventually replaced by a global oscillation of the entire forced medium. In any respect, however, this fact invalidates our claim of a pacemaker created out of noise to refer to the phenomenon at hand, since to strictly maintain the pacemaker structure we only need to think of an initial condition where the noisy forced excitable region is surrounded with a noise-free pure excitable area.

The supplementary, more quantitatively oriented, analysis of the phenomenon of excitable-oscillatory transitions referred to in this section, was aimed to compute the emitting period of the pacemaker. Results, summarized in Fig. 8, reproduce qualitatively the experimental observations for the chemical active Belousov-Zhabotinsky reaction reported in Ref. [13]. The transition between excitable and oscillatory conditions was estimated, in although reliable, yet not totally 

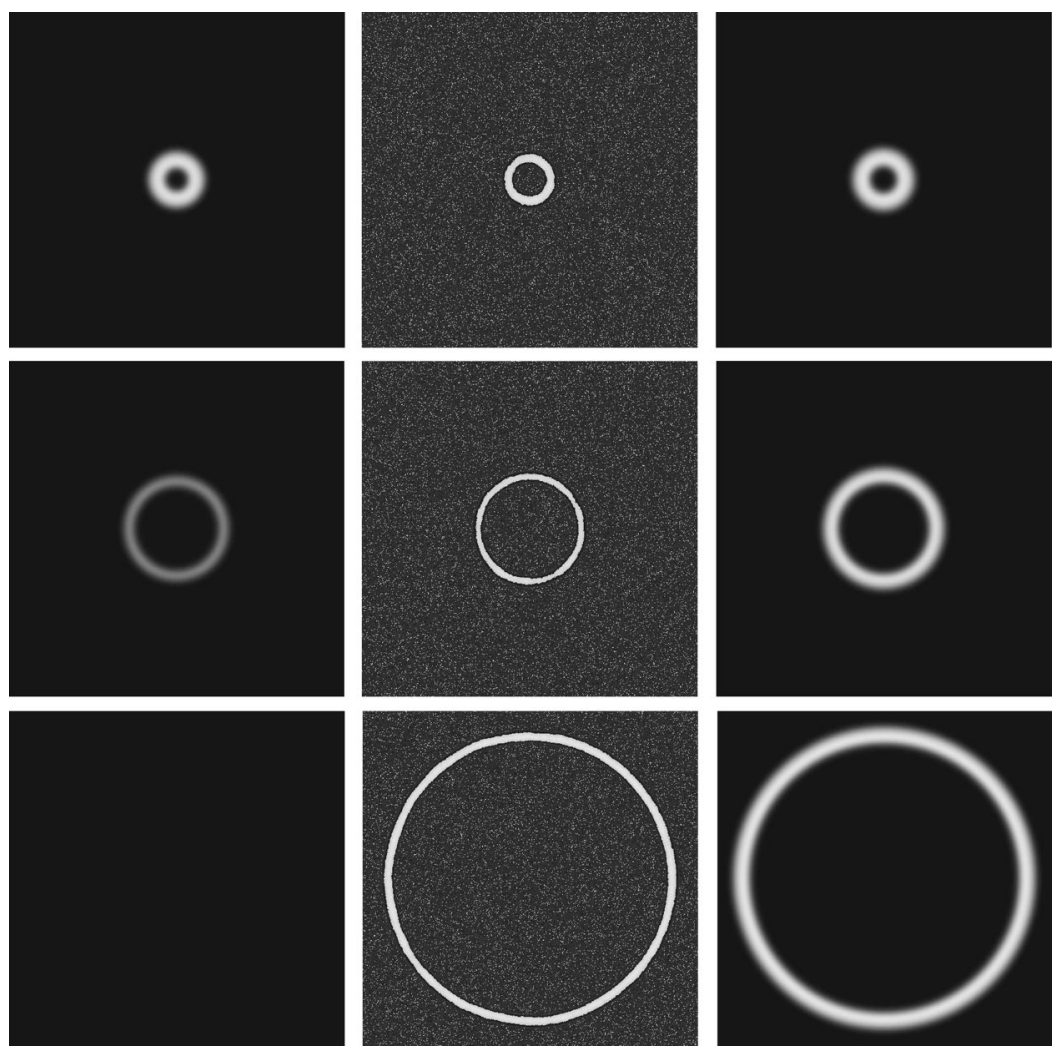

FIG. 3. First column: Propagation failure of an initial perturbation in a nonexcitable media free of fluctuations. Second column: Propagation of the same initial perturbation supported by stochastic fluctuations $\left(\sigma^{2}=0.00001\right)$. Third column: Propagation of the same initial perturbation for the effective deterministic model using the renormalized values of the parameters corresponding to the noise intensity $C(0)=0.001$. Time intervals correspond to 4.2 t.u. between the first and second rows and $12 \mathrm{t}$.u. between the second and third. Parameters of the model and of the simulations are: $a=0.7, b=0.07, \epsilon=0.04, \Delta x=0.1$ s.u., $\Delta t=0.001$ t.u., $512 \times 512$ pixels, and $D=1$ for this figure and the rest.

rigorous way, from the condition $b^{\prime}=0$ in Eq. (24). Note also with respect to our previous results for single front propagation, or even when we computed spiral rotation periods, that the agreement between the noisy and effective renormalized systems is a little bit worse for the oscillation frequency. This could be explained by a singular sensitivity of this later quantity to fluctuations as due to rare local nucleation effects. In any case, and as anticipated above in Sec. II, differences should be larger when increasing noise intensities. In spite of this small discrepancy, at the level of oscillation period, between the stochastic and the effective models, the agreement between the velocities of emitted waves (not shown) is again as remarkable as in Fig. 4. One final comment is worth mentioning here with respect to the periods plotted in Fig. 8. Presented results correspond to the time between the first and the second pulses. This time increases for the next pulses until it arrives the global oscillations period.

\section{SPATIOTEMPORAL STRUCTURED NOISE EFFECTS}

The preceding section was devoted to the study of different scenarios of transitions induced by uncorrelated fluctuation, i.e., exhibited by the emergence of sustained patterns of wave propagation purely arising from the intensity of a white noise approximation. Now we will concentrate on the effect of such external fluctuations on preexisting wave patterns. To gain generality and to prove that this behavior is by no means an artifact of using a white noise approximation, we will apply our general formalism, presented in Sec. II, to the case of generic structured spatiotemporal fluctuations. We do it in two steps: first we consider only temporal correlations and next we introduce also the spatial correlations.

\section{A. Time correlated noise}

As the most genuine parameter of wave propagation, we refer on what follows to the velocity of propagating waves as a function of the correlation time $(\tau)$, but keeping the noise white in space. The spatially white noise prescription in a lattice supposes that

$$
C(0)=\frac{\sigma^{2}}{\Delta x^{d}}, \quad C^{\prime \prime}(0)=-\frac{2^{d} \sigma^{2}}{\Delta x^{2+d}} .
$$

Quantitative results are summarized in Fig. 9 for different noise intensities and again contain both the simulations of the noisy system and those of the effective deterministic one. Now the main contribution to $\tau_{0}$ in Eq. (19) arises from the first term that is one order of magnitude greater than the 


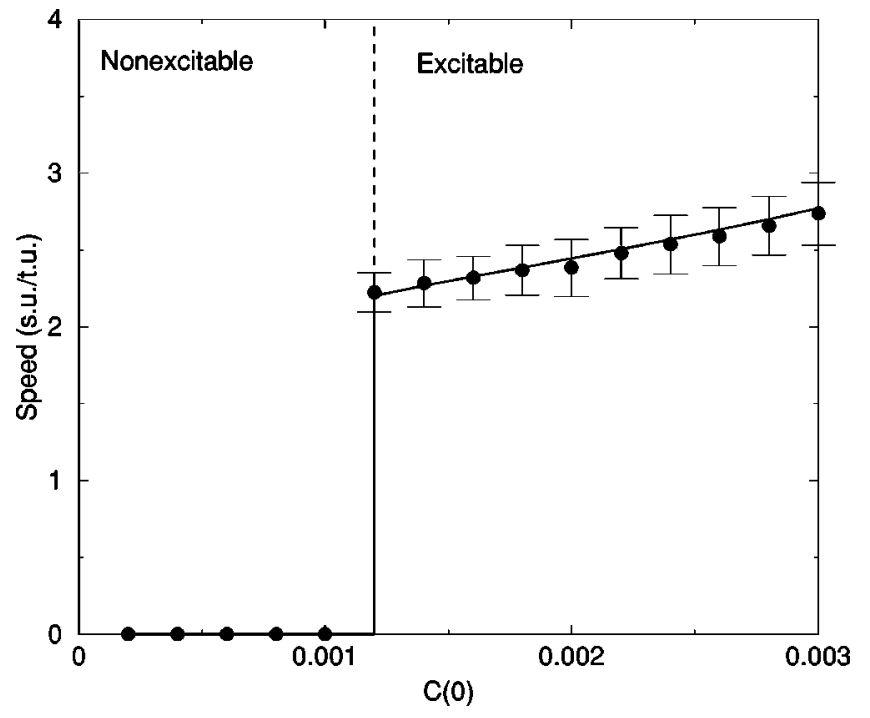

FIG. 4. Mean velocity of noise-supported traveling waves in a one-dimensional nonexcitable media vs the noise intensity, averaged with 25 realizations (points). Velocity of traveling wave for the effective deterministic model using the renormalized values of the parameters Eqs. (23)-(26) (solid line). Error bars correspond to the velocity dispersion. Vertical line shows the transition between different types of excitability. Parameters of the model and of the simulations are: $a=0.7, b=0.08, \epsilon=0.04, \Delta x=0.05$ s.u., $\Delta t$ $=0.0005$ t.u., and $L=100$ s.u. others ( $\tau_{0} \approx 0.0047$ t.u. for the system parameters of Fig. 9). Noting that the horizontal axis of Fig. 9 is logarithmic, one can see that the increase of the propagation speed is very sharp when $\tau$ approaches the new temporal scale introduced by the noise $\tau_{0}$.

It is important to realize how increasing the correlation time, and in a sense decreasing in this way the effective intensity of the fluctuations [see Eq. (26)], and in turn the excitability of the medium, the front velocity diminishes. For large correlation time the front speed for the averaged excitability is recovered. Note also the systematic effect observed for large correlation times $\tau$ in the one-dimensional stochastic simulations plotted in Fig. 9. Apparently the velocity is smaller than the deterministic value corresponding to the mean excitability. Something similar had been observed for dichotomic frozen noise in Ref. [19].

\section{B. Spatiotemporal correlated noise}

Here we add the parameter of spatial correlation length $\lambda$. Technical details can be found in the Appendix. Our simulations correspond now to two dimensional systems, with the explicit expressions for $C(0)$ and $C^{\prime \prime}(0)$ obtained from Eq. $(5)$

$$
C(0)=\frac{\sigma^{2}}{\pi \lambda^{2}}, \quad C^{\prime \prime}(0)=-\frac{\sigma^{2}}{\pi \lambda^{4}}
$$
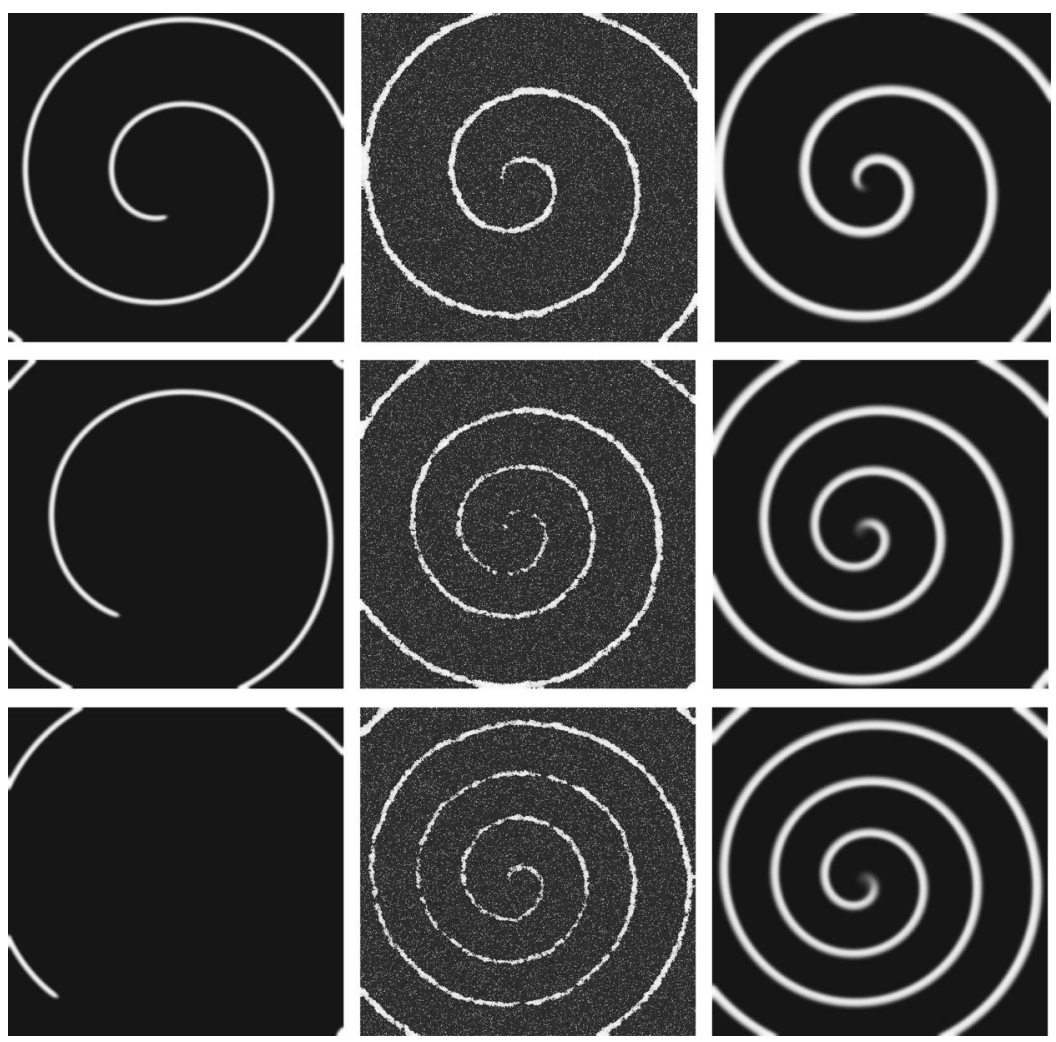

FIG. 5. First column: Propagation failure of a spiral wave in a subexcitable media free of fluctuations. Second column: Spiral wave in a subexcitable media sustained by stochastic fluctuations $\left(\sigma^{2}=0.0001\right)$. Third column: Spiral wave for the effective deterministic model using the renormalized values of the parameters corresponding to the noise intensity $C(0)=0.0025$. Time intervals correspond to 7.5 t.u. between each plot. Parameters of the model and of the simulations are: $a=0.7, b=0.13, \epsilon=0.02, \Delta x=0.2$ s.u., $\Delta t=0.005$ t.u., and $512 \times 512$ pixels. 


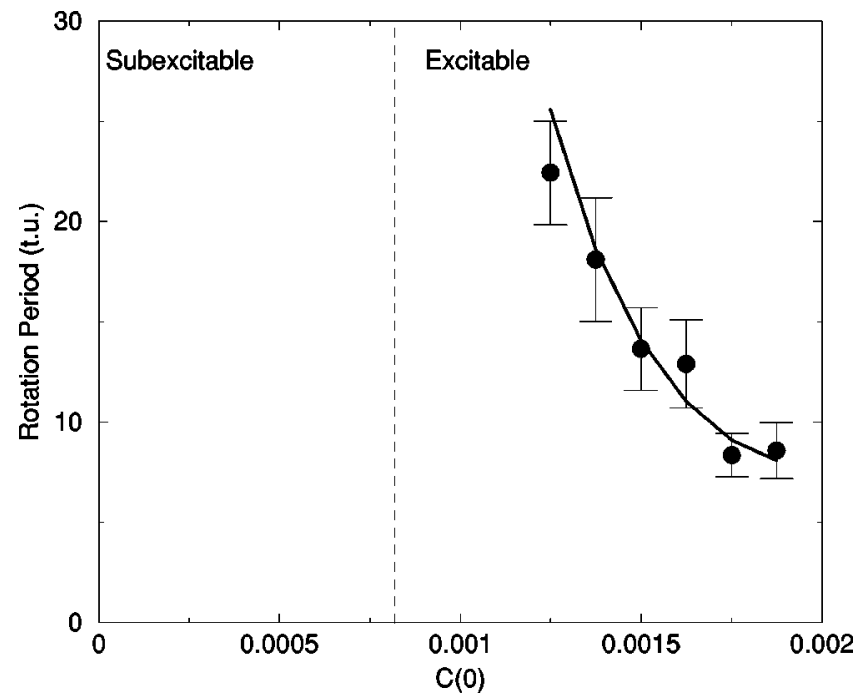

FIG. 6. Mean rotation period of noise-sustained spiral waves in a subexcitable media vs the noise intensity (points). Rotation period of the spiral wave for the effective deterministic model using the renormalized values of the parameters Eqs. (23)-(26) (solid line). Error bars correspond to the period dispersion. Vertical lines show the transition between different types of excitability. Parameters of the model and of the simulations are: $a=0.7, b=0.13, \epsilon$ $=0.02, \Delta x=0.2$ s.u., $\Delta t=0.005$ t.u., and $256 \times 256$ pixels.
Keeping the noise intensity $\sigma^{2}$ constant, increasing either the temporal $(\tau)$ or length $(\lambda)$ correlation parameters, the velocity of wave propagation decreases, as seen in Fig. 10. This is once more explained as a decrease in the effective intensity of the fluctuations [see Eqs. (26) and (28)]. Unlike the preceding section, we change the new temporal scale introduced by the noise $\tau_{0}$ when we modify the correlation length of the noise. In particular, the first term of Eq. (19) becomes increasingly irrelevant when $\lambda$ increases and then its effects are small (see caption of Fig. 10). These are the so called nontrivial effects of $\lambda$. Strong effects, although trivial, are those coming from the intensity of the noise, $C(0)$ $\sim \sigma^{2} / \lambda^{d}$, which can be absorbed as a renormalized intensity in a white noise assumption [Eq. (26)].

In Fig. 11 several front patterns for different values of the time and length correlation parameters are displayed. In all the situations, the front structure is largely maintained although it presents some unavoidable roughness induced by the stochastic nature of the forcing. This irregularity is more marked when decreasing both length and temporal correlation, although the correlation time effects seem more important within the temporal and spatial range of the fluctuations used in this study.

These three last figures clearly show how the noise parameters $\tau$ and $\lambda$ affect quantitatively the dynamics of chemical waves in excitable media under external fluctuations, and how these results are explained by our renormalized approach.
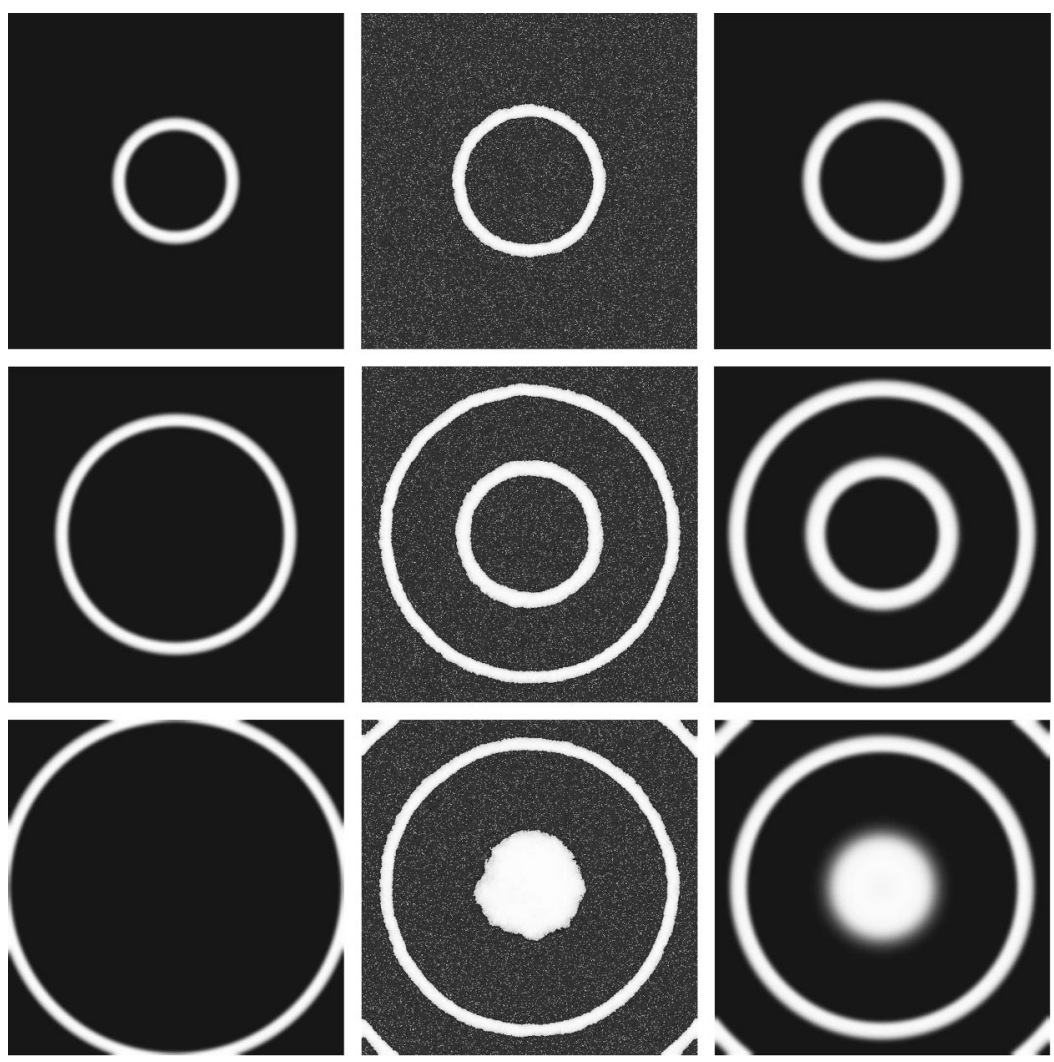

FIG. 7. First column: Propagation of a circular wave in an excitable media free of fluctuations. Second column: Propagation of a target pattern created out of stochastic fluctuations $\left(\sigma^{2}=0.00012\right)$. Third column: Propagation of target pattern for the effective deterministic model using the renormalized values of the parameters corresponding to the noise intensity $C(0)=0.003$. Time intervals correspond to 6 t.u. between each plot. Parameters of the model and of the simulations are: $a=0.7, b=0.02, \epsilon=0.04, \Delta x=0.2$ s.u., $\Delta \mathrm{t}=0.002$ t.u., and 512 $\times 512$ pixels. 


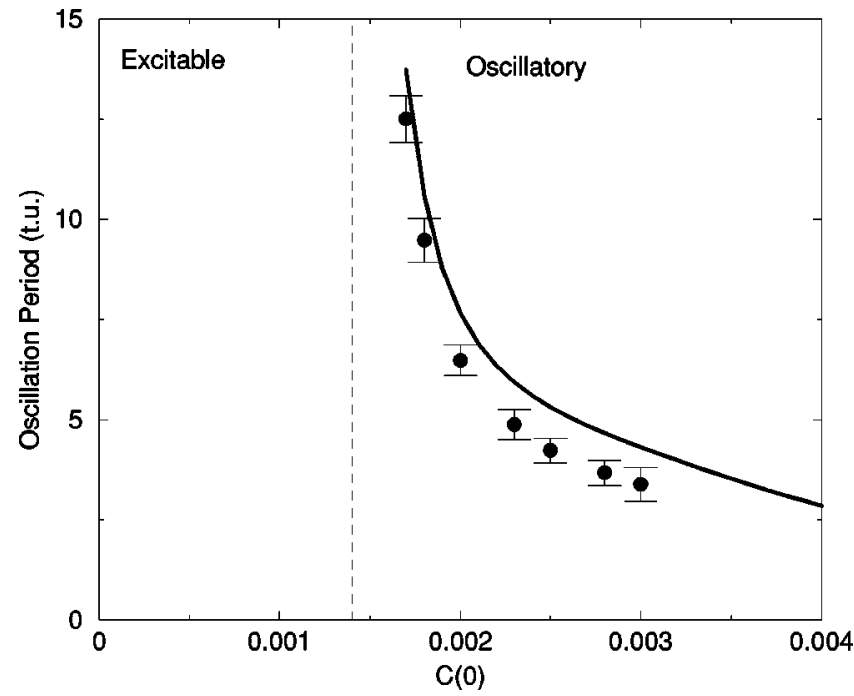

FIG. 8. Mean oscillation period of noise created wave trains in a one-dimensional subexcitable media vs the noise intensity, averaged with 25 realizations (points). Oscillation period of the wave train for the effective deterministic model using the renormalized values of the parameters Eqs. (23)-(26) (solid line). Error bars correspond to the period dispersion. Vertical lines show the transition between different types of excitability. Parameters of the model and of the simulations are: $a=0.7, b=0.1, \epsilon=0.02, \Delta x=0.05$ s.u., $\Delta t$ $=0.0005$ t.u., and $L=100$ s.u.

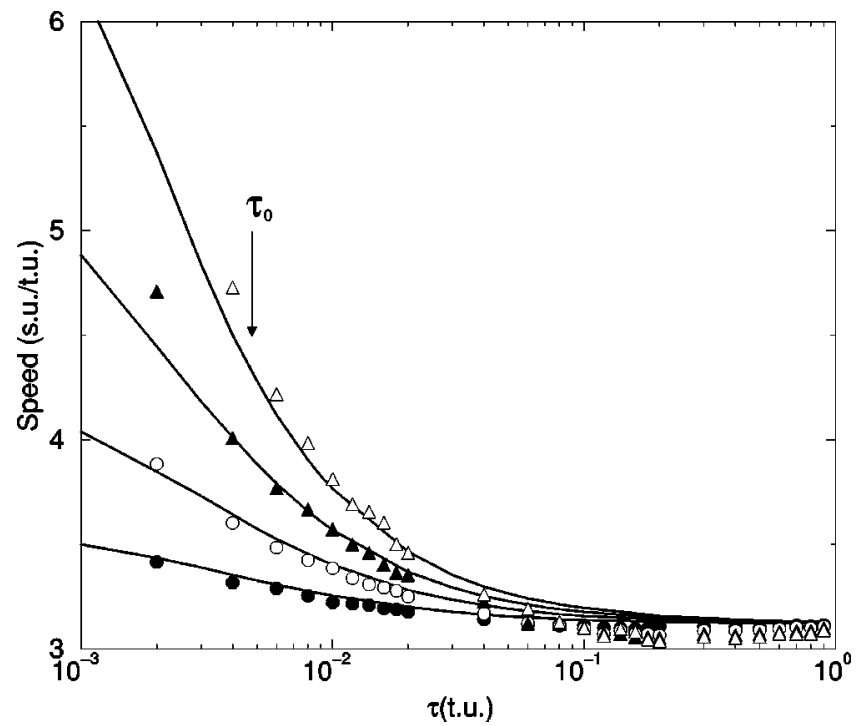

FIG. 9. Mean velocity of noisy traveling waves in a onedimensional excitable media vs the correlation time of the noise $(\tau)$ for different noise intensities: $\sigma^{2}=0.001$ (filled circles), $\sigma^{2}$ $=0.002$ (circles), $\sigma^{2}=0.003$ (filled triangles), and $\sigma^{2}=0.004$ (triangles). Velocity of traveling waves for the effective deterministic model using the renormalized values of the parameters, Eqs. (23)(26) (solid lines). Parameters of the model and of the simulations are: $a=0.7, b=0.1, \epsilon=0.02, \Delta x=0.1$ s.u., $\Delta t=0.001$ t.u., and $L$ $=400$ s.u.

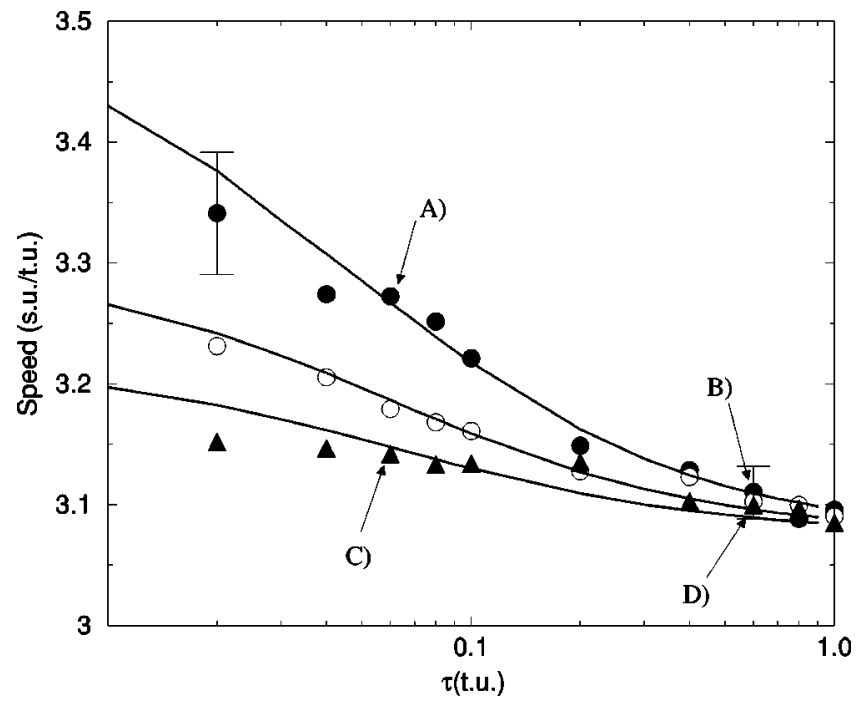

FIG. 10. Mean velocity of noisy traveling waves in a onedimensional excitable media vs the correlation time of the noise $(\tau)$, averaged with 25 realizations, for the same noise intensity $\left(\sigma^{2}=0.001\right)$ and for different correlation lengths: $\lambda=3 \Delta x$ s.u. (filled circles) $\quad\left(\tau_{0}=0.057\right.$ t.u. $), \lambda=4 \Delta x$ s.u. (circles) $\quad\left(\tau_{0}\right.$ $=0.061$ t.u.), and $\lambda=5 \Delta x$ s.u. (filled triangles) $\left(\tau_{0}=0.064\right.$ t.u.). Velocity of traveling waves for the effective deterministic model using the renormalized values of the parameters, Eqs. (23)-(25) (solid lines). For the sake of clarity only two characteristic error bars on the averaged velocity are shown. Labels (A)-(D) correspond to the cases in Fig. 11. Parameters of the model and of the simulations are: $a=0.7, b=0.1, \epsilon=0.02, \Delta x=0.2$ s.u., $\Delta t=0.005$ t.u., and $256 \times 256$ pixels.
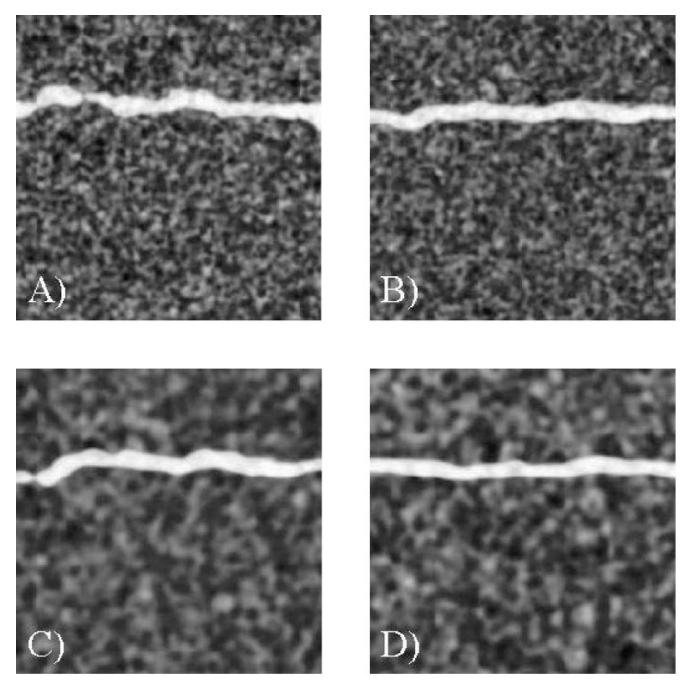

FIG. 11. Snapshots for two-dimensional fronts after $t$ $=11.25$ t.u. from the initial planar preparation, with the pattern of noise superposed for different values of the correlation time and correlation length and the same noise intensity $\left(\sigma^{2}=0.001\right)$ : (A) $\tau=0.06$ t.u. and $\lambda=3 \Delta x$ s.u. (mean velocity $v_{0}=3.23$ s.u./t.u.); (B) $\tau=0.6$ t.u. and $\lambda=3 \Delta x$ s.u. $\left(v_{0}=3.12\right.$ s.u./t.u. $)$; (C) $\tau$ $=0.06$ t.u. and $\lambda=5 \Delta x$ s.u. $\left(v_{0}=3.14\right.$ s.u./t.u. $)$; and (D) $\tau$ $=0.6$ t.u. and $\lambda=5 \Delta x$ s.u. $\left(v_{0}=3.08\right.$ s.u./t.u. $)$. Parameters of the model and of the simulations are: $a=0.7, b=0.1, \epsilon$ $=0.02, \Delta x=0.2$ s.u., $\Delta t=0.005$ t.u., and $256 \times 256$ pixels. 


\section{CONCLUSIONS}

We have developed an analytical approach for the evaluation of the systematic effects of spatiotemporal noises in activator-inhibitor equations. We have applied these calculations to the Barkley's model. We have analyzed separately the systematic effects of the different noise parameters $\sigma^{2}, \tau$, and $\lambda$. Increasing the noise intensity the excitability of the medium enhances giving rise to advanced transitions between different excitability regimes and change, correspondingly, the wave propagation characteristics. Nevertheless, if we increase either the temporal or the spatial correlations a decrease in the excitability is instead obtained.

Our main theoretical result is the renormalization of the parameters of the deterministic model, which allows us to predict and understand the noise effects by using only deterministic calculations and arguments. Numerical simulations of the stochastic system modeled by stochastic partial differential equations support our theoretical results with a high degree of accuracy.

\section{ACKNOWLEDGMENTS}

This work was supported by Dirección General de Investigación (DGI) and Comissionat per a Universitat i Recerca (Generalitat de Catalunya) under Project Nos. BXX20000638, BFM2000-0624, and 1999SGR00041. S.A. was financially supported by a FPI grant from Ministerio de Ciencia y Tecnología.

\section{APPENDIX}

We present here the numerical algorithm for the generation of noise with controlled spatiotemporal structure in a two-dimensional lattice. The numerical simulations corresponding to Secs. III (white noise in space and time) and IV A (white noise in space but correlated in time) have been performed with the usual algorithms [16]. Here we present details on the generation of both spatially and temporally correlated fluctuations.

The noise we have used through this work is a Gaussian process with zero mean and correlations given by Eq. (5).

A particular feature of the spatial correlation is that its
Fourier transform is also a Gaussian function,

$$
C(\mathbf{k})=\int_{\mathcal{R}} d x^{d} e^{-i \mathbf{k} \cdot \mathbf{x}} C(\mathbf{x})=e^{-\left(\lambda^{2} k^{2} / 2\right)}
$$

so, the noise correlation Eq. (4) in the Fourier space can be expressed as

$$
\left\langle\eta(\mathbf{k}, t), \eta\left(\mathbf{k}^{\prime}, t^{\prime}\right)\right\rangle=\sigma^{2} e^{-\left(\lambda^{2} k^{2} / 2\right)} \delta^{d}\left(\mathbf{k}+\mathbf{k}^{\prime}\right) \frac{1}{\tau} e^{-\left(\left|t-t^{\prime}\right| / \tau\right)} .
$$

The first step is to write the equation of motion of $\eta(\mathbf{k}, t)$,

$$
\tau \partial_{t} \eta=-\eta+\xi(\mathbf{k}, t)
$$

This equation is linear and a formal solution can be obtained in a straightforward way. Dividing the time in temporal steps, $\Delta t$, the formal solution can be written as [16]

$$
\eta(\mathbf{k}, t+\Delta t)=\eta(\mathbf{k}, t) e^{-\Delta t / \tau}+\sqrt{\frac{\sigma^{2}}{\tau}\left(1-e^{-2 \Delta t / \tau}\right)} \alpha(\mathbf{k}, t),
$$

where

$$
\alpha(\mathbf{k}, t)=[C(k)]^{1 / 2} \beta(\mathbf{k}, t)
$$

$\beta(\mathbf{k}, t)$ is a Gaussian white noise in Fourier space

$$
\left\langle\beta(\mathbf{k}, t), \beta\left(\mathbf{k}^{\prime}, t^{\prime}\right)\right\rangle=2 \delta_{t, t^{\prime}} \delta^{d}\left(\mathbf{k}+\mathbf{k}^{\prime}\right),
$$

which can be generated using standard procedures [20].

One can check that if the initial values of $\eta(\mathbf{k}, 0)$ are also Gaussian distributed with a correlation,

$$
\left\langle\eta(\mathbf{k}, 0), \eta\left(\mathbf{k}^{\prime}, 0\right)\right\rangle=2 \frac{\sigma^{2}}{\tau} e^{-\left(\lambda^{2} k^{2} / 2\right)} \delta^{d}\left(\mathbf{k}+\mathbf{k}^{\prime}\right),
$$

then the noise $\eta(\mathbf{k}, t)$ is a stationary, isotropic, and Gaussian stochastic process with correlation Eq. (A2). At each time integration step $\eta(\mathbf{k}, t)$ is generated and Fourier antitransformed to get $\eta(\mathbf{x}, t)$ that is used in the numerical algorithm to simulate Eq. (3). In this way the noise parameters $\sigma^{2}, \tau$, and $\lambda$ are controlled independently of each other.
[1] A. S. Mikhailov, Foundations of Synergetics-Distributed Active Systems (Springer-Verlag, Berlin, 1990).

[2] V. S. Zykov, Modeling of Wave Processes in Excitable Media (Manchester University Press, Manchester, 1988).

[3] R. Kapral and K. Showalter, Chemical Waves and Patterns (Kluwer, Dordrecht, The Netherlands, 1995).

[4] A. Goldbeter, Biochemical Oscillations and Cellular Rhythms (Cambridge University Press, Cambridge, UK, 1996).

[5] A. V. Panfilov and A. V. Holden, Computational Biology of the Heart (Wiley, Chichester, 1997).

[6] P. Jung, A. H. Cornell-Bell, K. Madden, and F. Moss, J. Neurophysiol. 79, 1098 (1998).

[7] J. Ross, S. C. Müller, and C. Vidal, Science 240, 460 (1988).

[8] A. T. Winfree, Science 175, 634 (1972).
[9] A. N. Zaikin and A. M. Zhabotinskii, Nature (London) 225, 535 (1970).

[10] A. T. Winfree and S. H. Strogatz, Nature (London) 311, 611 (1984); A. T. Winfree, SIAM Rev. 32, 1 (1990).

[11] J. F. Lindner, B. K. Meadows, W. L. Ditto, M. E. Inchiosa, and A. R. Bulsara, Phys. Rev. Lett. 75, 3 (1995).

[12] S. Kádár, J. Wang, and K. Showalter, Nature (London) 391, 770 (1998).

[13] S. Alonso, I. Sendiña-Nadal, V. Pérez-Muñuzuri, J. M. Sancho, and F. Sagués, Phys. Rev. Lett. 87, 078302 (2001).

[14] I. Sendiña-Nadal et al., Phys. Rev. Lett. 84, 2734 (2000).

[15] D. Barkley, M. Kness, and L. S. Tuckerman, Phys. Rev. A 42, 2489 (1990).

[16] J. García-Ojalvo and J. M. Sancho, Noise in Spatially Ex- 
tended Systems (Springer, New York, 1999).

[17] M. A. Santos and J. M. Sancho, Phys. Rev. E 64, 016129 (2001).

[18] J. García-Ojalvo and L. Schimansky-Geier, Europhys. Lett. 47,
298 (1999).

[19] I. Sendiña-Nadal et al., Phys. Rev. Lett. 80, 5437 (1998).

[20] J. García-Ojalvo, J. M. Sancho, and L. Ramírez-Piscina, Phys. Rev. A 46, 4670 (1992). 